\title{
La Sorcière de Marie NDiaye : du réalisme magique au banal invraisemblable
}

\author{
Andrée Mercier \\ Université Laval
}

À première vue, l'essentiel de l'œuvre romanesque de Marie NDiaye explore bien davantage les ressources de la fantaisie et de l'imaginaire que les modalités d'inscription du réel dans cet imaginaire. On y rencontre en effet une femme changée en bûche ${ }^{1}$, une jeune fille mangée par des chiens et qui reprendra vie comme si de rien n'était ${ }^{2}$, un homme dont l'épouse et le fils mystérieusement disparus réapparaissent sous les traits de fantômes ou d'âmes errantes ${ }^{3}$, ou encore un jeune homme

\footnotetext{
${ }^{1}$ La femme changée en bûche, 1989.

2 En famille, 1990.

3 Un temps de saison, 1994.
} 
constamment suivi par une bête indéfinissable, évoquant tout à la fois singe, oiseau et chien ${ }^{4}$. La forte présence du surnaturel ne situe pourtant pas les fictions de NDiaye dans le merveilleux, car un cadre de référence réaliste s'y déploie également. Les lieux, la temporalité, les personnages et les événements renvoient bien au monde contemporain et même, le plus souvent, à un quotidien des plus banals. Le roman La Sorcière (2003 [1996]), auquel je m'arrêterai ici plus particulièrement, est tout à fait exemplaire de cette manière. Le narrateur et personnage principal est une sorcière, prénommée Lucie, dotée de pouvoirs de divination. Comme toutes les femmes de sa lignée, Lucie initie ses filles à ce mystérieux pouvoir, mais elle le fait installée dans le sous-sol de son pavillon, vêtue d'un vieux jeans et chaussée de pantoufles, avant de retourner à la cuisine pour préparer les repas. Cela ne va pas sans mal. Non seulement ses deux filles et sa mère se révèlent beaucoup plus douées qu'elle pour la magie, mais Lucie se sent impuissante face aux aléas de sa vie : son mari la quitte, ses filles partent à leur tour, elle échoue à réconcilier ses parents, elle perd toutes ressources financières et se retrouvera même en prison ${ }^{5}$. Le but de mon étude sera de montrer, dans un premier temps, comment divers rapports au réel prennent forme dans ce roman, pour ensuite tenter de comprendre, plus précisément, à quoi tient un certain effritement du réel. Pour ce faire, j'avancerai l'hypothèse que cet effritement ne dépend pas principalement des éléments attestés de merveilleux ni de la problématisation du surnaturel par le fantastique, mais qu'il

\footnotetext{
${ }^{4}$ La femme changée en bûche.

5 Comme l'écrit W. Motte (2003) dans l'étude qu'il consacre à La Sorcière : « In short, Lucie's story would be an utterly banal one - were it not for the fact that she is a witch » (p. 115).
} 
dépend plutôt d'un invraisemblable qui prend sa source dans la narration même du banal et du quotidien. Il s'agira enfin de voir, brièvement, en quoi cet effritement et ce rapport au réel en quelque sorte démultiplié s'articulent autant à la déconstruction identitaire de Lucie qu'à une poétique de la fiction.

\section{Réalisme magique et fantastique}

La cohabitation du naturel et du surnaturel est constante dans La Sorcière. Si l'on reconnaît là un élément caractéristique du fantastique, c'est cependant sans l'hésitation, le doute ni l'antinomie qui habituellement l'accompagnent6. En effet, le surnaturel n'est pas contesté dans La Sorcière et rien n'est offert au lecteur pour tenter une explication rationnelle des événements extraordinaires qui s'y déroulent. Les filles de Lucie se métamorphoseront plus d'une fois en corneilles (voir entre autres p. 89-90, 98 et 118), sa mère transformera bel et bien son ancien mari en escargot (p. 151) et se montrera capable de surcroît d'apparaître dans un lieu et d'en disparaître tout aussi subitement (p. 110 et 116), et Lucie, quant à elle, versera des larmes de sang, signes qui accompagnent ses

\footnotetext{
${ }^{6}$ La réflexion sur le fantastique doit beaucoup à l'ouvrage de T. Todorov, Introduction à la littérature fantastique (1970), dont on mentionne souvent la citation suivante: «Le fantastique, c'est l'hésitation éprouvée par un être qui ne connaît que les lois naturelles, face à un événement en apparence surnaturel » (p. 29). Si Todorov fait de l'hésitation du lecteur la première condition du fantastique (p. 36), Charles W. Scheel (2005) insiste plutôt, à la suite de A. Chanady (Magical Realism and The Fantastic: Resolved Versus Unresolved Antinomy, New York et Londres, Garland Publ., 1985), sur l'antinomie entre deux niveaux de réalité - le naturel et le surnaturel antinomie qui reste irrésolue et qui, surtout, est issue de la façon dont le texte traite ces niveaux de réalité (p. 88-89).
} 
visions du passé, du présent et de l'avenir (notamment p. 23, $51,121,145)$. Il est vrai que c'est une sorcière elle-même qui raconte toute cette histoire et que l'on pourrait mettre en doute la fiabilité d'un tel narrateur. Néanmoins, aucun personnage de cet univers fictionnel ne vient faire valoir un point de vue rationnel sur les événements; et si les hommes sont généralement dégoûtés par le pouvoir des sorcières, leur dégoût traduit non pas un scepticisme mais, au contraire, une aversion pour un état de fait?

Globalement, c'est donc le mode du réalisme magique qui semble le mieux caractériser ce roman, puisque la cohabitation $\mathrm{du}$ naturel et du surnaturel n'y est pas conflictuelle. Le surnaturel n'est pas présenté ou ressenti comme problématique, bien qu'il s'inscrive dans un cadre réaliste ${ }^{8}$. En effet, Lucie est une jeune mère de famille aux prises avec des problèmes qui évoquent le monde actuel (éduquer des adolescentes, organiser sa vie après le départ de son mari) et elle évolue dans un univers où l'on reconnaît plusieurs enjeux

\footnotetext{
7 La réaction du gendarme, dans le passage suivant, illustre bien la réaction habituelle des hommes face à la sorcellerie : " Le gendarme s'était assis aussi loin de moi que possible et, lorsque je tournais les yeux vers lui pour me donner une contenance, son air rogue, défiant, prenait une fixité haineuse. Il me craignait, découvris-je avec étonnement. Il était jeune, grêle, craintif, et sa main avait répugné tout particulièrement à m'empoigner tout à l'heure » (p. 157).

8 J'emprunte à Scheel (2005) cette distinction du réalisme magique et du fantastique : "Alors que dans le fantastique, le surnaturel est perçu comme problématique, puisqu'il est manifestement antinomique par rapport au cadre rationnel du texte, le surnaturel dans le réalisme magique est accepté comme faisant partie de la réalité. Ce qui est antinomique au niveau sémantique est résolu au niveau de la fiction » (p. 90). Scheel ajoute que « [c]e qui est naturel ou surnaturel varie évidemment d'un lecteur à l'autre »; c'est donc « la voix narrative [...] qui indique au lecteur implicite si un événement doit être perçu ou non comme surnaturel » (p. 91).
} 
sociaux (la vie en banlieue, la situation de la femme, les différences générationnelles, le désir d'ascension sociale, etc.) ${ }^{9}$. Y sont aussi figurés avec précision les petits détails concrets de la vie domestique et familiale. C'est dans ce cadre que survient le surnaturel et que celui-ci est en somme admis comme faisant partie de la réalité. Il faut voir d'ailleurs avec quelle simplicité se trouvent réunies de bon matin à deviser sans plus de cérémonie la belle-mère et la mère de Lucie, alors que la seconde vient d'apparaître subitement dans la cuisine de l'autre!10

La cohabitation des deux cadres de référence, le naturel et le surnaturel, ne s'opère pas cependant sans certains glissements. Ainsi non seulement le merveilleux s'intègre-t-il dans un univers somme toute banal, mais se voit-il lui-même banalisé. Ce sont, par exemple, de bien modestes pouvoirs que ceux dont dispose Lucie. Ses visions sont toujours tronquées, n'offrant jamais de perspective d'ensemble, mais «que des détails sans importance, révélateurs de rien du tout » (NDiaye, 2001, 13). Elle n'y recourt d'ailleurs qu'assez peu, à la demande, par exemple, de sa voisine Isabelle préoccupée par l'avenir de

\footnotetext{
9 Dans son étude, W. Motte (2003) signale d'entrée de jeu la question de l'aliénation et de la marginalité qui caractérisent les divers personnages de l'œuvre de NDiaye : "While NDiaye's novels display a variety of settings and techniques, a common concern animates all of them, insofar as each is focused in some manner upon the questions of alienation and the problems experienced by an individual who finds herself or himself marginalized for reasons very largely beyond her or his understanding and control » (p. 115).

10 Comme le constate Lucie : «Elle [sa belle-mère] ne paraissait pas autrement surprise de la visite si matinale (ou si tardive, cette nuit?) de ma mère, qui, si mes souvenirs étaient exacts, mettait les pieds dans cette maison pour la première fois. Toutes deux s'étaient rencontrées à mon mariage treize ou quatorze ans auparavant, et jamais depuis lors. Elles échangèrent amabilités et lieux communs d'usage [...]» (p. 114).
} 
son fils Steve (p. 19-22), ou pour constater que son mari est en route pour la maison et qu'il lui reste encore un peu de temps pour préparer le dîner (p. 29). Bien que les pouvoirs de ses filles s'avèrent beaucoup plus puissants, c'est sans enthousiasme que ces dernières se prêtent à leur apprentissage, jugeant un tel don «fondamentalement inintéressant» (11) et de peu d'utilité dans la vie. Soulagées quand les leçons prennent fin, elles évoqueront leur initiation en déclarant : «Ce n'est pas pour dire, Maman, mais, vraiment, toutes ces conneries... » (p. 12). La mère de Lucie, pourtant si douée, refuse de faire usage de ses dons, sinon parfois en secret, préférant exercer, comme depuis 25 ans, le métier de secrétaire pour le compte d'une compagnie d'assurances. Comme l'affirme Lucie: «Personne, d'ailleurs, n'aurait pu ressembler moins à une sorcière que ma mère » (p. 67). Lucie, qui ne voit pas comment contrer le scepticisme de ses filles peu convaincues de la valeur pratique de leurs dons, se demande si le manque de foi de sa mère n'est pas en bonne partie responsable de ses « piètres capacités ». Enfin, non seulement ces dons apportentils peu à celles qui les possèdent, mais ils semblent plutôt nuire à la vie conjugale: le mari de Lucie éprouve un dégoût ostensible à l'égard des pouvoirs de sa femme, et la mère de Lucie a quitté son mari, sentant monter en elle une énergie violente qu'elle craignait de ne pouvoir contrôler. La banalisation du merveilleux est donc en bonne partie le fait des sorcières elles-mêmes qui, soit ont peu de talent pour la magie, soit éprouvent peu d'intérêt ou peu de conviction en leurs pouvoirs.

Curieusement, ce sont aussi les sorcières qui, dans ce récit dominé par le réalisme magique, feront surgir ponctuellement des moments de tension propre au fantastique. 
Devant l'apparition d'un corbeau à la fenêtre de sa cuisine, la mère de Lucie réagit avec violence comme s'il s'agissait là d'un événement inhabituel et alarmant. Lucie se demandera même avec inquiétude si ce n'est pas sa voisine Isabelle, femme ordinaire que rien n'associe pourtant à la sorcellerie, qui se cacherait ainsi sous les traits du volatile ${ }^{11}$. Alors que, jusque-là, le surnaturel prenait place sans soubresaut dans le récit, il devient tout à coup étrange aux yeux mêmes des sorcières qui portent ici un regard rationnel et inquiet sur le merveilleux. Ces moments demeureront rares dans la trame du récit, mais ils viennent déstabiliser l'articulation des cadres de référence mis en place par le réalisme magique, en créant une antinomie entre le naturel et le surnaturel.

\section{Un banal invraisemblable}

Alors que le surnaturel se voit banalisé, le banal, lui, prend un air étrange. Si, bien sûr, on peut voir là l'effet quasi inévitable de la présence du merveilleux et, plus encore, d'éléments de fantastique qui viendraient en quelque sorte contaminer le cadre réaliste, c'est néanmoins la narration même du banal et

\footnotetext{
11 C'est ce que montre, parmi d'autres, le passage suivant : «Un grand oiseau brun se tenait sur le rebord [de la fenêtre], nous observant à travers la vitre d'un œil vigilant et sans effroi, si semblable à la corneille que j'avais remarquée le matin même qu'un petit frisson d'inquiétude me parcourut. / Un corbeau! s'écria ma mère. / Alors je ne pus m'empêcher de chuchoter en direction de l'oiseau : / -Isabelle? / Mais ma mère ouvrait la fenêtre avec fracas et, soudain furieuse, elle que je ne connaissais que d'humeur égale, conciliante, agita les mains en clamant : / - Veux-tu te sauver, sale bête! Ouh, ouh, déguerpis! [...] Elle referma prudemment la fenêtre. Lorsqu'elle revint vers nous, elle était rouge, en colère, déconfite. Jamais je ne l'avais vue ainsi hors d'elle et toute violence à grand-peine contenue. Je lui pris la main, déconcertée de si peu la reconnaître » (p. 79-80).
} 
du quotidien qui semble ici en cause. Plusieurs éléments peuvent être signalés. On observera premièrement que le récit se déploie en glissant d'une histoire à l'autre, et d'une thématique à une autre, esquissant chaque fois une possible intrigue aussitôt abandonnée. Par exemple, le début du récit campe d'emblée le monde de la sorcellerie et semble devoir explorer la singularité de ce monde occulte : «Quand mes filles eurent atteint l'âge de douze ans, je les initiai aux mystérieux pouvoirs » (p. 9). Cependant, l'initiation aux pouvoirs cède le pas à la vie familiale, aux rapports qu'entretient Lucie avec ses filles et, surtout, avec son mari qui, peu après, quittera le domicile conjugal. La rupture elle-même ne retient toutefois pas l'attention du récit et devient secondaire. Lucie décidera plutôt de réconcilier ses parents, séparés depuis cinq ans. Puis, à la demande de son père, elle s'efforcera de retrouver une importante somme d'argent qu'elle avait reçue comme avance sur son héritage, argent avec lequel s'est sauvé son mari. Et ainsi de suite. Si l'univers familial reste toujours une thématique centrale, la trame narrative, elle, se construit, on le voit, par une série d'abandons ou de changements de direction, qui remettent chaque fois en question la quête de Lucie. Les possibles programmes narratifs se succèdent, tombent à plat ou encore s'amorcent sans motivation apparente.

Ainsi en va-t-il du désir de Lucie de réconcilier ses parents, qui constitue malgré tout la quête la plus soutenue parmi les petites intrigues avortées du récit. Lucie y voit une entreprise absolument nécessaire et en affirme plus d'une fois l'importance sans toutefois vraiment l'expliquer. C'est d'ailleurs d'une façon bien singulière, sinon contradictoire, qu'elle énonce le caractère vital de cette quête: "Si mes parents devaient rester séparés, il est probable que je n'y survivrais pas, pensais- 
je vaguement»(p. 96). Cette quête apparaît d'autant plus déraisonnable que Lucie ne cherche jamais à retrouver son propre mari qui vient pourtant de la quitter, et que ce projet s'interrompt de façon plutôt cocasse quand le père se voit transformé en escargot.

Ce que l'on constate en observant la trame générale du récit se trouve encore plus nettement dans le détail de l'enchaînement événementiel. En effet, l'excès et la soudaineté marquent avec une fréquence remarquable tout le déroulement du récit. Cela donne à l'articulation des actions et des états une allure syncopée, sans grande logique. Je cite ici et là : «j'ouvris soudain la fenêtre » (p. 22), « elle se leva brusquement » (p. 22), «l'épuisement et la honte m'accablèrent d'un seul coup » (p. 26), « soudain un peu fatigué » (p. 27), « subitement gênée » (p. 37), « j'eus envie soudain » (p. 37), « tout d'un coup » (p. 37), «soudain de mauvaise humeur» (p. 43), «la figure [...] brutalement bouffie et décomposée » (p. 43), "la soudaine roideur de son buste»(p. 44), etc. Les personnages agissent «brusquement», «brutalement», «vivement», «bruyamment»; ils surgissent, se saisissent, s'emparent, s'écrient. Les transformations psychologiques et physiques se font de manière aussi radicale et brutale que les métamorphoses de sorcières en corneilles : Lucie a du mal à reconnaître son père devenu coquet et peu scrupuleux, elle constate le changement de sa jeune belle-sœur devenue grosse et pleine d'assurance ; de même, la belle-mère de Lucie ne reconnaît plus ses enfants, devenus violents avec elle. Les transformations sont innombrables, subites, extrêmes et toujours inexpliquées. Elles suscitent d'ailleurs l'étonnement, la peur ou un certain malaise chez ceux qui les constatent. 
Bien des coïncidences viennent par ailleurs ponctuer le récit, ce qui accentue encore le caractère gratuit ou saugrenu de l'enchaînement événementiel. Ainsi en est-il de ses voisins, Isabelle et Steve, que Lucie, curieusement, rencontre souvent lors de ses déplacements (c'est le cas p. 54, 70-71 et 131); ou de son mari Pierrot, accompagné de sa mère et de sa nouvelle famille, qui surgissent inopinément à la fin du récit (p. 162); coïncidences fort à propos que le récit ne tente même pas de justifier.

On peut mentionner enfin le cadre temporel du récit qui apparaît de plus en plus problématique au fil de l'histoire. Déjà quelque peu ébranlée par les très nombreuses marques aspectuelles de soudaineté qui ponctuent le détail de la chaîne événementielle, la temporalité se montre de moins en moins vraisemblable, notamment en ce qui concerne la durée de l'histoire racontée. Plutôt rares, les indications temporelles semblent néanmoins ramener la durée de la séquence événementielle à une étendue de quelques mois (peut-être quatre ou cinq), c'est-à-dire de la fin de l'hiver au début des vacances d'été12. Si la première partie du récit correspond à une durée plausible (en quelques semaines, Lucie voit partir son mari, puis elle se rend à Paris et à Poitiers pour de très brefs séjours), la seconde partie échappe à une motivation réaliste. Ainsi, en quelques semaines à peine, Pierrot, le mari de Lucie, se retrouve bien installé au sein d'une nouvelle famille, une vision de Lucie le montrant « en père de famille qui rentre chez lui comme chaque soir depuis des années» (p. 122). Surtout, Isabelle, cette voisine envahissante et désœuvrée, confinée

12 On peut aussi ramener la durée de la séquence événementielle à un peu plus d'un an, si l'on inclut la période de l'initiation aux pouvoirs rapportée au début du récit. 
depuis des années dans son quartier à la périphérie d'une petite ville, est tout à coup à la tête d'une université féminine, lucrative affaire qu'elle a elle-même montée, ayant su convaincre

les différentes instances administratives du département et de la région d'avancer les fonds importants qu'il lui avait fallu pour la location des bureaux et de l'internat, pour l'engagement des professeurs et du personnel, pour les pages de publicité dans la presse locale. (p. 134)

Or cette université qui fonctionne rondement n'existait pas, faut-il le préciser, un mois plus tôt!

À considérer les événements ordinaires de la vie de Lucie (c'est-à-dire en excluant les métamorphoses, visions et autres phénomènes surnaturels), on constate donc une narration qui vient miner la vraisemblance et déstabiliser le cadre réaliste du récit, plus fortement encore que ne le font les éléments attestés de merveilleux et les quelques moments de tension fantastique. Autrement dit, aux entorses à la vraisemblance empirique (relative à l'expérience commune) s'ajoute une déstabilisation constante de la mise en intrigue, qui concerne plutôt la vraisemblance diégétique ${ }^{13}$. Le rapport au réel se voit ainsi démultiplié dans La Sorcière puisqu'on voit y cohabiter réalisme magique, fantastique et invraisemblance, et que chacun semble déconstruire la perspective adoptée par l'autre. Le réalisme magique tend à résoudre l'antinomie qui oppose naturel et surnaturel et «facilite ainsi l'acceptation de l'incongru» (Scheel, 2005: 91), sans inféoder toutefois le

\footnotetext{
13 J'emprunte la distinction vraisemblance empirique et vraisemblance diégétique à Cécile Cavillac (1995). S'ajoute un troisième terme, la vraisemblance pragmatique, qui concerne cette fois la crédibilité du narrateur et de la situation énonciative.
} 
surnaturel au code réaliste (Scheel, 2005 : 114). Le fantastique, au contraire, maintient une tension entre le naturel et le surnaturel, et conserve sa préséance au cadre rationnel à l'aune duquel on juge le surnaturel inacceptable. Enfin, l'invraisemblable, qui surgit dans les strictes limites d'un cadre rationnel et réaliste ${ }^{14}$, déstabilise le naturel mais sans recourir pour cela au surnaturel; l'inexplicable et l'étonnant résidant dans le naturel lui-même.

\section{Le sens du réel et de la fiction}

Cette multiplication des codes et des rapports entre ces codes entraîne, on en conviendra, un certain effritement du réel et de sa représentation. Il y a pourtant un sens aigu du réel dans $L a$ Sorcière, expression que j'emprunte à Jacques Dubois (2000), dans la mesure où il y a malgré tout figuration d'un monde et de l'expérience d'un sujet dans le monde ${ }^{15}$. À travers la subjectivité et le parcours de Lucie, le récit raconte la perte graduelle de repères identitaires. Épouse, mère, fille, voisine, sorcière, Lucie

14 Précisons que l'usage du terme naturel, dans son opposition au terme surnaturel, ne signifie aucunement l'idée d'une nature dégagée de toute dimension culturelle.

15 Je renvoie plus particulièrement au premier chapitre de l'ouvrage de Dubois, chapitre intitulé «Le sens du réel » (p. 27-45) qui, s'il porte sur le roman réaliste, offre de belles pistes de réflexion pour aborder d'autres esthétiques romanesques comme le montre le passage suivant : « [...] l'idée de sens du réel peut se décliner de bien des façons. Elle est par exemple attention à tout le contexte matériel et culturel qui entoure les êtres et les définit. Ce qui implique une sensibilité au monde dans ce qu'il a de plus concret et de plus effectif. [...] Elle est encore sens de la vie en mouvement, de la durée dans sa substance, de la vie psychique dans ses transformations. Enfin, ce sens est celui du social. Pour l'écrivain du réel, tout univers est socialisé, c'est-à-dire que le destin individuel, qui reste la ligne de faîte du romanesque, ne prend valeur et relief qu'au sein d'une vie collective et d'un écheveau de relations » (p. 28-29). 
n'est plus rien de tout cela au terme du récit: son mari l'a quittée, ses filles également, son père a disparu, ses liens avec sa mère sont coupés, elle n'a plus de maison, et, ironie du sort, on l'accuse même de se prétendre frauduleusement sorcière. Comme bien des personnages de l'œuvre de Marie NDiaye, Lucie est de plus en plus marginalisée. Elle semble condamnée à une altérité et à une impuissance qui rendent impossible son inscription dans le monde. Ce monde est violent, il n'offre pas de règles claires, il est fondamentalement incompréhensible. Ce monde, de plus, n'est pas le même pour tous : il y a ceux qui s'étonnent et ceux qui ne s'étonnent de rien. On l'a vu, la démultiplication des rapports au réel contribue fortement à la représentation de ce monde hétérogène, imprévisible, insaisissable, peuplé de personnages qui tentent tant bien que mal d'y prendre place ou d'en sortir. La figure de la sorcière recouvre d'ailleurs fort bien le lien qui unit le rapport au réel et la marginalité, c'est-à-dire le double statut de Lucie, à la fois étrangère et étrange ${ }^{16}$.

Ce sens du réel ne crée pas une fiction confortable pour le lecteur, privé lui aussi de ses repères et placé devant un monde qui, à la fois, évoque et ébranle un horizon de référence connu. On aurait tort toutefois d'engager trop strictement le roman de

\footnotetext{
16 Sur ce point les propos de Teko-Agbo, cités par W. Motte, bien qu'ils portent sur un autre roman de NDiaye, En famille, sont particulièrement éloquents : " Here is where Marie NDiaye's fiction stands out. She valorizes by revealing the problem of the strange and invites us to reflect on the place of the strange and the stranger in our societies. With what eyes do we look upon the stranger? » K. Ambroise Teko-Agbo, "En famille or the Problem of Alterity », Research in African Literatures, vol. 26, No. 2 (Summer 1995), p. 163; cité par W. Motte (2003 : 133). Dans un essai qu'il consacre à l'œuvre de NDiaye, Mathieu Lindon (2004) emprunte à NDiaye elle-même le terme "étrangéité » qu'elle utilise pour désigner le décalage ontologique de ses personnages (p. 38-39).
} 
NDiaye dans une entreprise de figuration de la réalité individuelle et sociale. D'autant plus que, à travers la figure de la sorcellerie et le jeu des rapports au réel, se déploie, à petites touches et non sans ironie, une poétique de l'imaginaire qui n'a de cesse de questionner les limites et les usages de la fiction ${ }^{17}$. Entre le pouvoir de divination de Lucie, qui ne permet de voir que l'insignifiant (NDiaye, 2003, p. 13), et celui de sa mère ou de ses filles capable des plus grands prodiges, se met en scène une cartographie des territoires de la fiction, du minimalisme aux possibles flamboyances du merveilleux. Mais c'est peut-être plus encore une réflexion sur le pacte d'adhésion à la fiction qui se voit mise en scène. Le piètre don de Lucie, qui ne lui permet de voir que "des détails sans importance», «la couleur d'un habit, l'aspect du ciel, une tasse de café fumant» (13), ne s'explique-t-il pas après tout par le manque de foi de sa mère en ces pouvoirs immémoriaux? La survie du don n'est-elle pas compromise, par ailleurs, par l'indifférence de ses filles qui n'y voient aucune utilité pratique, au contraire des feuilletons télévisés dont elles sont férues et qui leur semblent offrir la connaissance des comportements humains (53)?

Au cœur de La Sorcière se voit donc posé un problème de croyance en la sorcellerie et par là aussi - et surtout - en la fiction. Le monde dans lequel évolue Lucie n'adhère plus au

\footnotetext{
17 Dans un essai qu'il consacre à NDiaye, Ook Chung (1994) voit dans l'œuvre un questionnement de la littérature: "Cette métaphore de la création littéraire et, de manière extensive, de toute construction herméneutique, traverse presque tous les romans » (p. 162). Les romans qui se sont ajoutés depuis (l'essai de Chung datant de 1994) vont dans le même sens, La Sorcière accusant plus fortement encore une dimension métafictionnelle notamment par sa dominante magico-réaliste qui brouille, entre autres, les limites génériques et les catégories esthétiques. On lira, sur cet aspect du réalisme magique, la présentation du dossier « $\mathrm{Du}$ fantastique réel au réalisme magique » de la revue Textyles (Denis, 2002).
} 
fabuleux. Les pouvoirs des sorcières suscitent le dégoût ou le désintérêt. Tout comme les filles de Lucie et sa voisine Isabelle, ce monde est avide de connaissances tangibles et d'avantages concrets. Or, de l'aveu même de Lucie, elle ne peut offrir à ses filles aucun exemple de situation où son don lui aurait rendu service ou apporté quelque bénéfice (p. 13). La question de l'adhésion se double donc ici d'un enjeu véridictoire et d'une axiologie: dans le monde de La Sorcière, on croit vrai ce qui paraît utile. Si les sorcières elles-mêmes peinent à croire à l'utilité de leur don, nombreux pourtant sont les dupes: les belles rentrées d'argent de Pierrot, capable de faire croire aux promesses de vacances idylliques du Garden-Club (p. 29-30), et les gains que rapportent à Isabelle les savoirs ésotériques de son université féminine de la santé spirituelle ${ }^{18}$, montrent qu'il est facile de se laisser prendre aux jeux de l'apparence.

Y a-t-il encore une place dans ce monde moderne pour les sorcières? Le roman de Marie NDiaye laisse la question en suspens. Au terme de son parcours, Lucie aura tout perdu, si ce n'est une certaine foi dans les pouvoirs occultes de la lignée de femmes dont elle provient et la conviction qu'il faut à tout prix s'efforcer de transmettre ce don. Quant au récit lui-même, il aura exploré bien des modalités de la fiction, obligeant son lecteur à constamment renégocier les cadres de référence et le pacte d'adhésion qui le lient à cette histoire de sorcières.

18 Comme l'affirme Isabelle à Lucie : « Quel boniment, si tu savais! [...] Mais il n'y a rien qui paie mieux, en ce moment » (p. 137). 


\section{Bibliographie}

CAVILLAC, Cécile (1995), "Vraisemblance pragmatique et autorité fictionnelle », Poétique, n 101 (février), p. 23-46.

CHUNG, Ook (1994), «Lire en français. Le Roman de l'absence », Liberté, no 216 (décembre), p. 157-166.

DENIS, Benoît (dir.) (2002), Textyles (dossier « Du fantastique réel au réalisme magique »), n 21, Bruxelles, Éd. Le Cri.

DUBOIS, Jacques (2000), Les Romanciers du réel. De Balzac à Simenon, Paris, Seuil, coll. « Points ».

LINDON, Mathieu (2004), Je vous écris. Récits critiques, Paris, P.O.L.

MOTTE, Warren (2003), Fables of the Novel. French Fiction Since 1990, Champaign (Illinois), Dalkey Archive Press.

NDIAYE, Marie (1989), La Femme changée en bûche, Paris, Éditions de Minuit;

- (1990), En Famille, Paris, Éditions de Minuit;

- ([1994] 2004), Un Temps de saison, suivi de La Trublionne de Pierre Lepage, Paris, Éditions de Minuit, coll. « Double » 28;

- ([1996] 2003), La Sorcière, Paris, Éditions de Minuit, coll. « Double » 21.

SCHEEL, Charles W. (2005), Réalisme magique et réalisme merveilleux, Des Théories aux poétiques, Paris, L'Harmattan.

TODOROV, Tzvetan (1970), Introduction à la littérature fantastique, Paris, Seuil, coll. « Poétique». 


\title{
Résumé
}

Représentatif de l'œuvre de NDiaye, le roman La sorcière (Minuit, 1996) semble multiplier les rapports au réel: merveilleux, fantastique, réalisme magique, quotidien et banalité y cohabitent. La narratrice et personnage principal est une sorcière, prénommée Lucie, dotée de pouvoirs de divination somme toute modestes, habitant dans un "petit lotissement de pavillons neufs". Mère de deux filles qui se révéleront beaucoup plus douées qu'elle pour la magie, elle voit sa vie et son identité lui échapper peu à peu : son mari et ses filles la quittent, elle échoue à réconcilier ses parents, elle perd toutes ressources financières et se retrouvera même en prison. Le but de notre étude est de montrer comment divers rapports au réel prennent forme dans ce roman et, plus particulièrement, comment l'effritement du réel tient moins aux éléments attestés de merveilleux (la présence de sorcières, de métamorphoses et d'apparitions), ou à une problématisation du surnaturel par le fantastique, qu'à un invraisemblable qui prend précisément sa source dans le banal et le quotidien. Il s'agira ensuite de voir en quoi ce rapport au réel démultiplié s'articule autant à la déconstruction identitaire de Lucie qu'à une poétique de la fiction.

\begin{abstract}
Representative of the work of NDiaye, the novel La sorcière (The Witch) (Minuit, 1996) appears to multiply the ties with reality: fantasy, magical realism, routine and banality all coexist on the same level of relevance. The narrator and protagonist is a witch named Lucie, who has modest powers of divination and lives in a "small subdivision of new bungalows". Being the mother of two young girls (more gifted as witches than herself), she is slowly losing the control of her life and identity: her
\end{abstract}


husband and daughters are leaving her, she attempts and fails to reconcile her estranged parents, she loses her home and savings before ending up in jail. The objective of this study will be to demonstrate how different connections with reality coexist in this novel and more particularly, how this crumbling of reality is less the consequence of the presence of attested elements of fantasy (witches, metamorphosis and apparitions) or of the problematization of supernatural and fantasy, than from the unlikelihood coming from the banality and routine. Then, the demonstration will be focused on how this multitude of rapports to reality can articulate, at the same time, the destruction of Lucie's identity as well as the construction of an esthetic of fiction. 